\title{
Research Article \\ Compact Composition Operators between Bloch Type Spaces in the Polydisk
}

\author{
Zhong-Shan Fang ${ }^{1,2}$ \\ ${ }^{1}$ Department of Mathematics, Tianjin University, Tianjin 300072, China \\ ${ }^{2}$ Department of Mathematics, Tianjin Polytechnic University, Tianjin 300387, China \\ Correspondence should be addressed to Zhong-Shan Fang, fangzhongshan@yahoo.com.cn
}

Received 11 September 2012; Accepted 9 October 2012

Academic Editor: Jinhu Lü

Copyright (C) 2012 Zhong-Shan Fang. This is an open access article distributed under the Creative Commons Attribution License, which permits unrestricted use, distribution, and reproduction in any medium, provided the original work is properly cited.

Wulan et al. (2009), Wulan et al. characterized the compactness of composition operators on the Bloch space in the unit disk by the $n$th power of the induced analytic function. This paper will generalize the result to the Bloch type space in the polydisk.

\section{Introduction}

Let $\mathbb{D}^{n}$ be the polydisk of $\mathbb{C}^{n}$ with boundary $\partial \mathbb{D}^{n}$. The class of all holomorphic functions on the domain $\mathbb{D}^{n}$ will be denoted by $H\left(\mathbb{D}^{n}\right)$. Let $\varphi(z)=\left(\varphi_{1}(z), \ldots, \varphi_{n}(z)\right)$ be a holomorphic self-map of $\mathbb{D}^{n}$. The composition operator is defined as follows:

$$
C_{\varphi}(f)(z)=f(\varphi(z))
$$

for any $f \in H\left(\mathbb{D}^{n}\right)$ and $z \in \mathbb{D}^{n}$.

The study of the composition operator dates back to the late 60s. From then on, the boundedness and compactness of composition operators between several spaces of holomorphic functions have been studied extensively. We refer the interested readers to the books in [1-3]. Recently, there has been lot of work for Bloch type spaces. For example, see [4-9], as well as the related references therein. There are still many unsolved problems of interest to numerous mathematicians. 
For $p>0$, the Bloch type space $\mathbb{B}^{p}$ consists of those $f \in H\left(\mathbb{D}^{n}\right)$ such that

$$
\|f\|_{p}=|f(0)|+\sup _{z \in \mathbb{D}^{n}} \sum_{k=1}^{n}\left(1-\left|z_{k}\right|^{2}\right)^{p}\left|\frac{\partial f}{\partial z_{k}}(z)\right|<\infty ;
$$

with this norm, it becomes a Banach space. When $p=1$, it is the classical Bloch space.

In [10], Wulan et al. obtained a new result about the compactness of the composition operators on the Bloch space in the unit disk. We state it as follows.

Theorem 1.1. Let $\varphi$ be an analytic self-map of the unit disk $\mathbb{D}$. Then $C_{\varphi}$ is compact on the Bloch space if and only if

$$
\lim _{m \rightarrow \infty}\left\|\varphi^{m}\right\|_{1}=0
$$

where $\varphi^{m}$ here means the mth power of $\varphi$.

Along with the further research, it is natural to consider the higher-dimensional case. The goal of this paper is to extend the above result in the unit disk to the polydisk.

Throughout this paper, let $\mathbb{N}$ be the set of the positive integers, $I=\left\{i \in \mathbb{N}:\left\|\varphi_{i}\right\|_{\infty}=1\right\}$ and $J=\left\{j \in \mathbb{N}:\left\|\varphi_{j}\right\|_{\infty}<1\right\}$.

\section{Some Lemmas}

In this section, we present some lemmas which will be used in the proofs of our main results in the next section.

The proof of the following lemma can be found in the proof of Theorem 1.1 in [8], see also Lemma 2.2 in [7].

Lemma 2.1. Let $p>0, m \in \mathbb{N}$ and $0 \leq x \leq 1$. And let $H_{m, p}(x)=x^{m-1}\left(1-x^{2}\right)^{p}, r_{m}=$ $((m-1) /(m-1+2 p))^{1 / 2} \quad(m \geq 2)$. Then for $m \geq 2, H_{m, p}(x)$ is decreasing on $\left[r_{m}, r_{m+1}\right]$, and

$$
\lim _{m \rightarrow \infty} m^{p} \min _{x \in\left[r_{m}, r_{m+1}\right]} H_{m, p}(x)=\left(\frac{2 p}{e}\right)^{p}
$$

Lemma 2.2 (see Corollary 3, [6]). Let $p \geq 1$ and $q>0$. Suppose that $\varphi=\left(\varphi_{1}, \ldots, \varphi_{n}\right)$ is a holomorphic self-map of $\mathbb{D}^{n}$. Then $C_{\varphi}: \mathbb{B}^{p} \rightarrow \mathbb{B}^{q}$ is compact if and only if

(1) $\varphi_{l}(z) \in \mathbb{B}^{q}, l \in\{1, \ldots, n\}$;

(2) $\lim _{\varphi(z) \rightarrow \partial \mathbb{D}^{n}} \sum_{k, l=1}^{n}\left|\left(\partial \varphi_{l} / \partial z_{k}\right)(z)\right|\left(1-\left|z_{k}\right|^{2}\right)^{q} /\left(1-\left|\varphi_{l}(z)\right|^{2}\right)^{p}=0$.

Lemma 2.3 (see Corollary 4, [6]). Let $0<p<1$ and $q>0$. Suppose that $\varphi=\left(\varphi_{1}, \ldots, \varphi_{n}\right)$ is a holomorphic self-map of $\mathbb{D}^{n}$. Then $C_{\varphi}: \mathbb{B}^{p} \rightarrow \mathbb{B}^{q}$ is compact if and only if

(3) $C_{\varphi}$ is bounded;

(4) $\lim _{\left|\varphi_{l}(z)\right| \rightarrow 1} \sum_{k, l=1}^{n}\left|\left(\partial \varphi_{l} / \partial z_{k}\right)(z)\right|\left(1-\left|z_{k}\right|^{2}\right)^{q} /\left(1-\left|\varphi_{l}(z)\right|^{2}\right)^{p}=0, l \in\{1, \ldots, n\}$. 
The following lemma is the crucial criterion for the compactness of $C_{\varphi}$, whose proof is an easy modification of the proof of Proposition 3.11 in [1].

Lemma 2.4. Assume that $\varphi$ is a holomorphic self-map of $\mathbb{D}^{n}$. Then $C_{\varphi}: \mathbb{B}^{p} \rightarrow \mathbb{B}^{9}$ is compact if and only if $C_{\varphi}$ is bounded and for any bounded sequence $\left\{f_{m}\right\}_{m \in \mathbb{N}}$ in $\mathbb{B}^{p}$ which converges to zero uniformly on compact subsets of $\mathbb{D}^{n}$, we have

$$
\left\|C_{\varphi} f_{m}\right\|_{q} \longrightarrow 0
$$

as $m \rightarrow \infty$.

\section{Main Theorems}

In $[6,8]$, the authors characterized the boundedness and compactness of composition operators between different Bloch type spaces in the polydisk. In this section, we will give some new results about the old problems.

Theorem 3.1. Let $p \geq 1$ and $q>0$. Suppose that $\varphi=\left(\varphi_{1}, \ldots, \varphi_{n}\right)$ is a holomorphic self-map of $\mathbb{D}^{n}$. Then $C_{\varphi}: \mathbb{B}^{p} \rightarrow \mathbb{B}^{q}$ is compact if and only if

(5) $\varphi_{l}(z) \in B^{q}, l \in\{1, \ldots, n\}$;

(6) $\lim _{m \rightarrow \infty} m^{p-1}\left\|\varphi_{i}^{m}\right\|_{q}=0, i \in I$;

(7) $\lim _{\varphi(z) \rightarrow \partial \mathbb{D}^{n}} \sum_{k=1}^{n}\left|\left(\partial \varphi_{j} / \partial z_{k}\right)(z)\right|\left(1-\left|z_{k}\right|^{2}\right)^{q}=0, j \in J$.

Proof. For any $l \in\{1, \ldots, n\}$, set

$$
a_{l}=\lim _{\varphi(z) \rightarrow \partial \mathbb{D}^{n}} \sum_{k=1}^{n}\left|\frac{\partial \varphi_{l}}{\partial z_{k}}(z)\right| \frac{\left(1-\left|z_{k}\right|^{2}\right)^{q}}{\left(1-\left|\varphi_{l}(z)\right|^{2}\right)^{p}} .
$$

For the proof of the sufficiency, by Lemma 2.2, we only have to show that $a_{l}=0$ for every $l \in\{1,2, \ldots, n\}$. There are two cases to consider.

Case 1. If $j \in J$ with $\left\|\varphi_{j}\right\|_{\infty}<1$, we have

$$
\lim _{\varphi(z) \rightarrow \partial \mathbb{D}^{n}} \sum_{k=1}^{n}\left|\frac{\partial \varphi_{j}}{\partial z_{k}}(z)\right|\left(1-\left|z_{k}\right|^{2}\right)^{q} \leq a_{j} \leq \lim _{\varphi(z) \rightarrow \partial \mathbb{D}^{n}} \sum_{k=1}^{n}\left|\frac{\partial \varphi_{j}}{\partial z_{k}}(z)\right| \frac{\left(1-\left|z_{k}\right|^{2}\right)^{q}}{\left(1-\left\|\varphi_{j}\right\|_{\infty}^{2}\right)^{p}} .
$$

Therefore, for any $j \in J, \lim _{\varphi(z) \rightarrow \partial \mathbb{D}^{n}} \sum_{k=1}^{n}\left|\left(\partial \varphi_{j} / \partial z_{k}\right)(z)\right|\left(1-\left|z_{k}\right|^{2}\right)^{q}=0$ is equivalent to $a_{j}=0$. Case 2. If $i \in I$ with $\left\|\varphi_{i}\right\|_{\infty}=1$, then for each $m \geq 2$, let

$$
A_{m, i}=\left\{z=\left(z_{1}, \ldots, z_{n}\right) \in \mathbb{D}^{n}: r_{m} \leq\left|z_{i}\right| \leq r_{m+1}\right\},
$$

where $r_{m}=((m-1) /(m-1+2 \alpha))^{1 / 2}$. 
For each fixed $i \in I$ and every $\varepsilon>0$, there exists a $\delta_{0}$ with $0<\delta_{0}<1$ such that

$$
\sum_{k=1}^{n}\left|\frac{\partial \varphi_{i}}{\partial z_{k}}(z)\right| \frac{\left(1-\left|z_{k}\right|^{2}\right)^{q}}{\left(1-\left|\varphi_{i}(z)\right|^{2}\right)^{p}}>a_{i}-\varepsilon
$$

whenever $\operatorname{dist}\left(\varphi(z), \partial \mathbb{D}^{n}\right)<\delta_{0}$.

Since $r_{m} \rightarrow 1$ as $m \rightarrow \infty$, we may choose sufficiently large $m$ such that $r_{m}>1-\delta_{0}$. If $\varphi(z) \in A_{m, i}$, then

$$
\begin{gathered}
r_{m} \leq\left|\varphi_{i}(z)\right| \leq r_{m+1}, \\
1-r_{m+1}<1-\left|\varphi_{i}(z)\right|<1-r_{m}<\delta_{0}
\end{gathered}
$$

thus

$$
\operatorname{dist}\left(\varphi_{i}(z), \partial \mathbb{D}\right)<\delta_{0}
$$

There exists $w_{i}$ with $\left|w_{i}\right|=1$ such that

$$
\operatorname{dist}\left(\varphi_{i}(z), w_{i}\right)=\operatorname{dist}\left(\varphi_{i}(z), \partial \mathbb{D}\right)<\delta_{0}
$$

Let $w=\left(\varphi_{1}(z), \ldots, \varphi_{i-1}(z), w_{i}, \varphi_{i+1}(z), \ldots, \varphi_{n}(z)\right)$. Then

$$
\operatorname{dist}\left(\varphi(z), \partial \mathbb{D}^{n}\right) \leq \operatorname{dist}(\varphi(z), w) \leq \operatorname{dist}\left(\varphi_{i}(z), w_{i}\right)<\delta_{0} .
$$

So we have

$$
\sup _{\varphi(z) \in A_{m, i}} \sum_{k=1}^{n}\left|\frac{\partial \varphi_{i}}{\partial z_{k}}(z)\right| \frac{\left(1-\left|z_{k}\right|^{2}\right)^{q}}{\left(1-\left|\varphi_{i}(z)\right|^{2}\right)^{p}}>a_{i}-\varepsilon
$$


Letting $\varepsilon \rightarrow 0$ and by Lemma 2.1, we have

$$
\begin{aligned}
a_{i} & \leq \lim _{m \rightarrow \infty} \sup _{\varphi(z) \in A_{m, i}} \sum_{k=1}^{n}\left|\frac{\partial \varphi_{i}}{\partial z_{k}}(z)\right| \frac{\left(1-\left|z_{k}\right|^{2}\right)^{q}}{\left(1-\left|\varphi_{i}(z)\right|^{2}\right)^{p}} \\
& \leq \lim _{m \rightarrow \infty} \sup _{\varphi(z) \in A_{m, i}} \sum_{k=1}^{n}\left|\frac{\partial \varphi_{i}}{\partial z_{k}}(z)\right| \frac{m^{p}\left|\varphi_{i}\right|^{m-1}\left(1-\left|z_{k}\right|^{2}\right)^{q}}{m^{p}\left|\varphi_{i}\right|^{m-1}\left(1-\left|\varphi_{i}(z)\right|^{2}\right)^{p}} \\
& \leq \frac{\lim _{m \rightarrow \infty} \sup _{\varphi(z) \in A_{m, i}} \sum_{k=1}^{n}\left|\left(\partial \varphi_{i} / \partial z_{k}\right)(z)\right| m^{p}\left|\varphi_{i}\right|^{m-1}\left(1-\left|z_{k}\right|^{2}\right)^{q}}{\lim _{m \rightarrow \infty} \inf _{\varphi(z) \in A_{m, i}} m^{p}\left|\varphi_{i}\right|^{m-1}\left(1-\left|\varphi_{i}(z)\right|^{2}\right)^{p}} \\
& \leq\left(\frac{e}{2 p}\right)^{p} \lim _{m \rightarrow \infty} m^{p-1}\left\|\varphi_{i}^{m}\right\|_{q} .
\end{aligned}
$$

From which and (6), we know $a_{i}=0$. Combining the two cases, thus (2) holds. Note that conditions (5) and (1) are the same, it follows from Lemma 2.2 that $C_{\varphi}: \mathbb{B}^{p} \rightarrow \mathbb{B}^{q}$ is compact.

Now we turn to prove the necessity. The result (5) follows by Lemma 2.2. Using Lemma 2.1, we see that

$$
\lim _{m \rightarrow \infty} m^{p-1}\left\|z_{l}^{m}\right\|_{p}=\left(\frac{2 p}{e}\right)^{p}
$$

for any $l \in\{1, \ldots, n\}$.

For any $i \in I$, we consider the test functions $f_{m}\left(z_{1}, z_{2}, \ldots, z_{n}\right)=z_{i}^{m} /\left\|z_{i}^{m}\right\|_{p}$. It is clear that $\left\|f_{m}\right\|_{p}=1$ and $f_{m} \rightarrow 0$ uniformly on compact subsets of $\mathbb{D}^{n}$ as $m \rightarrow \infty$. If $C_{\varphi}$ is compact, then

$$
\begin{aligned}
0 & =\lim _{m \rightarrow \infty}\left\|C_{\varphi} f_{m}\right\|_{q}=\lim _{m \rightarrow \infty} \frac{\left\|\varphi_{i}^{m}\right\|_{q}}{\left\|z_{i}^{m}\right\|_{p}} \\
& =\lim _{m \rightarrow \infty} \frac{m^{p-1}\left\|\varphi_{i}^{m}\right\|_{q}}{m^{p-1}\left\|z_{i}^{m}\right\|_{p}} \\
& =\left(\frac{e}{2 p}\right)^{p} \lim _{m \rightarrow \infty} m^{p-1}\left\|\varphi_{i}^{m}\right\|_{q} .
\end{aligned}
$$

This shows that (6) holds.

For any $j \in J$, from the discussion of Case 1 in the proof of the sufficiency, it follows that $a_{j}=0$. And this fact implies that condition (7) holds. Now the proof of the theorem is completed.

Remark 3.2. For $n=1$, if $p=q=1$, it is well known that $C_{\varphi}$ is always bounded on the Bloch space, which implies that (5) is true. Note that in this case, conditions (6) and (7) are the same. Thus, we immediately obtain the result on the Bloch space in [10] by Theorem 3.1. 
Theorem 3.3. Let $0<p<1$ and $q>0$. Suppose that $\varphi=\left(\varphi_{1}, \ldots, \varphi_{n}\right)$ is a holomorphic self-map of $\mathbb{D}^{n}$. Then $C_{\varphi}: \mathbb{B}^{p} \rightarrow \mathbb{B}^{q}$ is compact if and only if

(8) $C_{\varphi}$ is bounded;

(9) $\lim _{m \rightarrow \infty} m^{p-1}\left\|\varphi_{i}^{m}\right\|_{q}=0, i \in I$.

Proof. Suppose first that $C_{\varphi}: \mathbb{B}^{p} \rightarrow \boldsymbol{B}^{q}$ is compact. It is clear that $C_{\varphi}: \mathbb{B}^{p} \rightarrow \mathbb{B}^{q}$ is bounded. Taking the test functions $f_{m}(z)=z_{i}^{m} /\left\|z_{i}^{m}\right\|_{p}$, and using the same arguments as in the proofs of Theorem 3.1, we obtain

$$
\lim _{m \rightarrow \infty} m^{p-1}\left\|\varphi_{i}^{m}\right\|_{q}=0
$$

for any $i \in I$.

This proves the necessity.

Conversely, by Lemma 2.4, it suffices to show that (4) holds. In fact, for any $j \in J$ with $\left\|\varphi_{j}\right\|_{\infty}<1$, there exists some positive number $\delta$ close enough to 1 , such that the set $E_{j}=\left\{z \in \mathbb{D}^{n}:\left|\varphi_{j}(z)\right|>\delta\right\}$ is empty. Without loss of generality, we may assume that

$$
\lim _{\left|\varphi_{j}(z)\right| \rightarrow 1} \sum_{k=1}^{n}\left|\frac{\partial \varphi_{j}}{\partial z_{k}}(z)\right| \frac{\left(1-\left|z_{k}\right|^{2}\right)^{q}}{\left(1-\left|\varphi_{j}(z)\right|^{2}\right)^{p}}=0 .
$$

For any $i \in I$, and $m \geq 2$, let

$$
A_{m, i}=\left\{z=\left(z_{1}, \ldots, z_{n}\right) \in \mathbb{D}^{n}: r_{m} \leq\left|z_{i}\right| \leq r_{m+1}\right\},
$$

where $r_{m}=((m-1) /(m-1+2 \alpha))^{1 / 2}$.

Therefore, we have

$$
\begin{aligned}
& \lim _{\left|\varphi_{i}(z)\right| \rightarrow 1} \sum_{k=1}^{n}\left|\frac{\partial \varphi_{i}}{\partial z_{k}}(z)\right| \frac{\left(1-\left|z_{k}\right|^{2}\right)^{q}}{\left(1-\left|\varphi_{i}(z)\right|^{2}\right)^{p}} \\
& \quad \leq \lim _{m \rightarrow \infty} \sup _{\varphi(z) \in A_{m, i}} \sum_{k=1}^{n}\left|\frac{\partial \varphi_{i}}{\partial z_{k}}(z)\right| \frac{\left(1-\left|z_{k}\right|^{2}\right)^{q}}{\left(1-\left|\varphi_{i}(z)\right|^{2}\right)^{p}} \\
& \quad \leq \lim _{m \rightarrow \infty} \sup _{\varphi(z) \in A_{m, i}} \sum_{k=1}^{n}\left|\frac{\partial \varphi_{i}}{\partial z_{k}}(z)\right| \frac{m^{p}\left|\varphi_{i}\right|^{m-1}\left(1-\left|z_{k}\right|^{2}\right)^{q}}{m^{p}\left|\varphi_{i}\right|^{m-1}\left(1-\left|\varphi_{i}(z)\right|^{2}\right)^{p}} \\
& \quad \leq \frac{\lim _{m \rightarrow \infty} \sup _{\varphi(z) \in A_{m, i}} \sum_{k=1}^{n}\left|\left(\partial \varphi_{i} / \partial z_{k}\right)(z)\right| m^{p}\left|\varphi_{i}\right|^{m-1}\left(1-\left|z_{k}\right|^{2}\right)^{q}}{\lim _{m \rightarrow \infty} \inf _{\varphi(z) \in A_{m, i}} m^{p}\left|\varphi_{i}\right|^{m-1}\left(1-\left|\varphi_{i}(z)\right|^{2}\right)^{p}} \\
& \quad \leq\left(\frac{e}{2 p}\right)^{p} \lim _{m \rightarrow \infty} m^{p-1}\left\|\varphi_{i}^{m}\right\|_{q} \cdot
\end{aligned}
$$


This along with condition (9) yields that

$$
\lim _{\left|\varphi_{i}(z)\right| \rightarrow 1} \sum_{k=1}^{n}\left|\frac{\partial \varphi_{i}}{\partial z_{k}}(z)\right| \frac{\left(1-\left|z_{k}\right|^{2}\right)^{q}}{\left(1-\left|\varphi_{i}(z)\right|^{2}\right)^{p}}=0
$$

for any $i \in I$.

Combining the results of the two cases for $j \in J$ and $i \in I$, we get (4). By Lemma 2.3, we know that $C_{\varphi}: \mathbb{B}^{p} \rightarrow \mathbb{B}^{q}$ is compact. This completes the proof of the theorem.

\section{Acknowledgment}

The authors would like to thank the referees for the useful comments and suggestions which improved the presentation of this paper.

\section{References}

[1] C. C. Cowen and B. D. MacCluer, Composition Operators on Spaces of Analytic Functions, CRC Press, Boca Raton, Fla, USA, 1994.

[2] J. H. Shapiro, Composition Operators and Classical Function Theory, Springer, New York, NY, USA, 1993.

[3] K. H. Zhu, Operator Theory in Function Spaces, vol. 139, Marcel Dekker, New York, NY, USA, 1990.

[4] K. Madigan and A. Matheson, "Compact composition operators on the Bloch space," Transactions of the American Mathematical Society, vol. 347, no. 7, pp. 2679-2687, 1995.

[5] J. H. Shapiro, "The essential norm of a composition operator," Annals of Mathematics, vol. 125, no. 2, pp. 375-404, 1987.

[6] S. Stević, R. Y. Chen, and Z. H. Zhou, "Weighted composition operators between Bloch-type spaces in the polydisk," Sbornik Mathematics, vol. 201, no. 2, 2010.

[7] R. H. Zhao, "Essential norms of composition operators between Bloch type spaces," Proceedings of the American Mathematical Society, vol. 138, no. 7, pp. 2537-2546, 2010.

[8] Z. H. Zhou and Y. Liu, "The essential norms of composition operators between generalized Bloch spaces in the polydisc and their applications," Journal of Inequalities and Applications, vol. 2006, Article ID 90742, 22 pages, 2006.

[9] Z. H. Zhou and J. H. Shi, "Compact composition operators on the Bloch space in polydiscs," Science in China Series A, vol. 44, no. 3, pp. 286-291, 2001.

[10] H. C. Wulan, D. C. Zheng, and K. H. Zhu, "Compact composition operators on BMOA and the Bloch space," Proceedings of the American Mathematical Society, vol. 137, no. 11, pp. 3861-3868, 2009. 


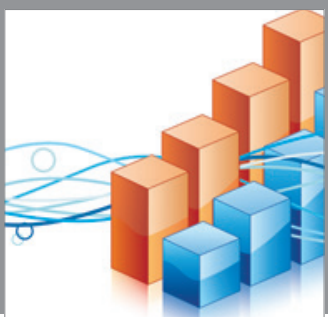

Advances in

Operations Research

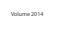

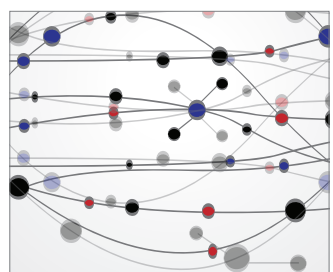

\section{The Scientific} World Journal
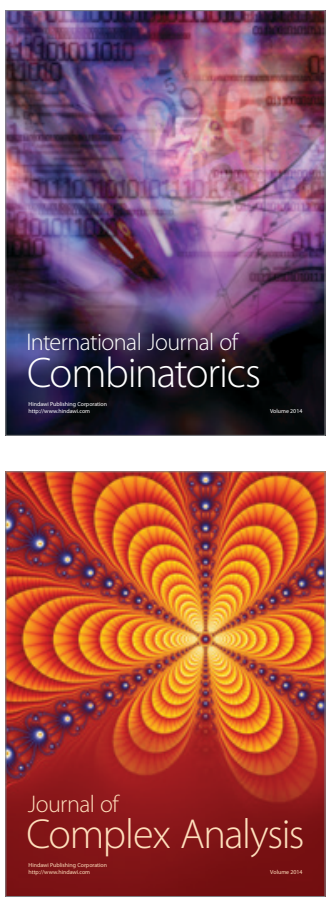

International Journal of

Mathematics and

Mathematical

Sciences
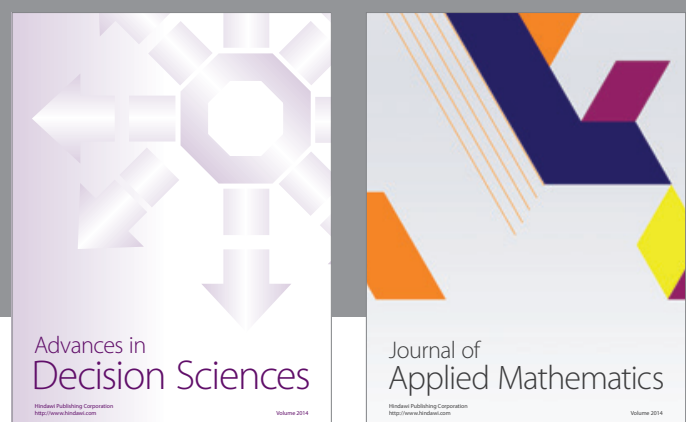

Journal of

Applied Mathematics
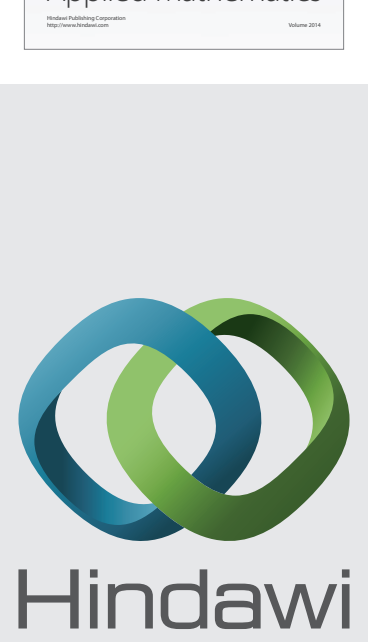

Submit your manuscripts at http://www.hindawi.com
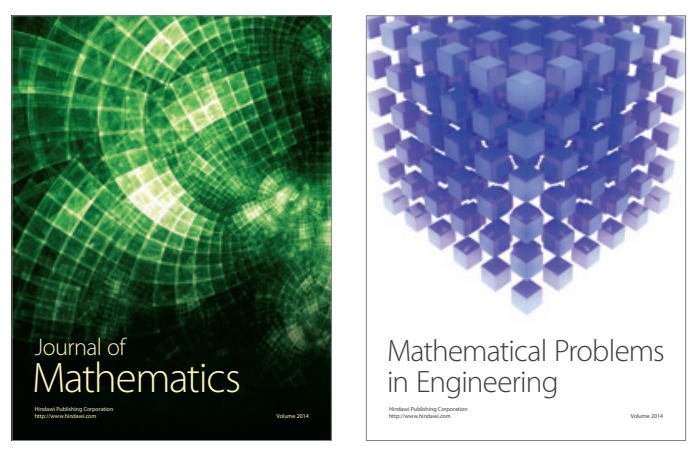

Mathematical Problems in Engineering
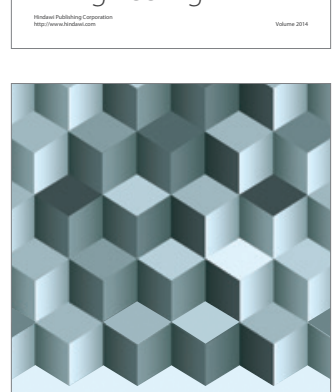

Journal of

Function Spaces
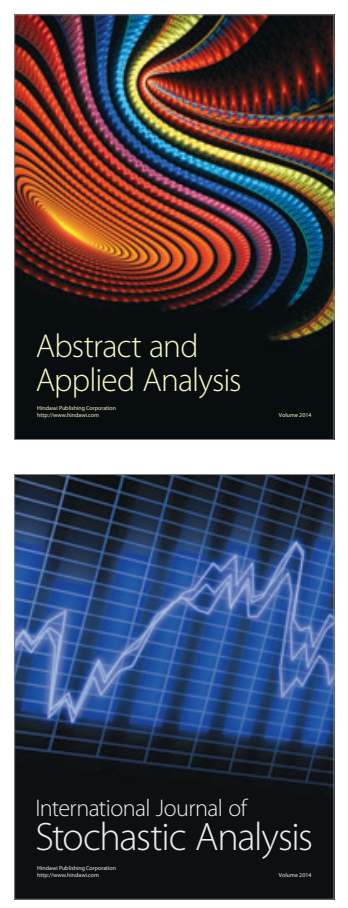

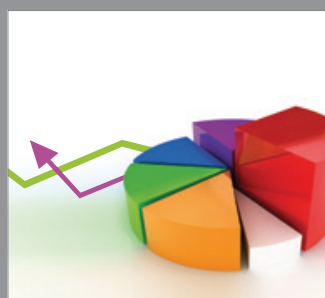

ournal of

Probability and Statistics

Promensencen
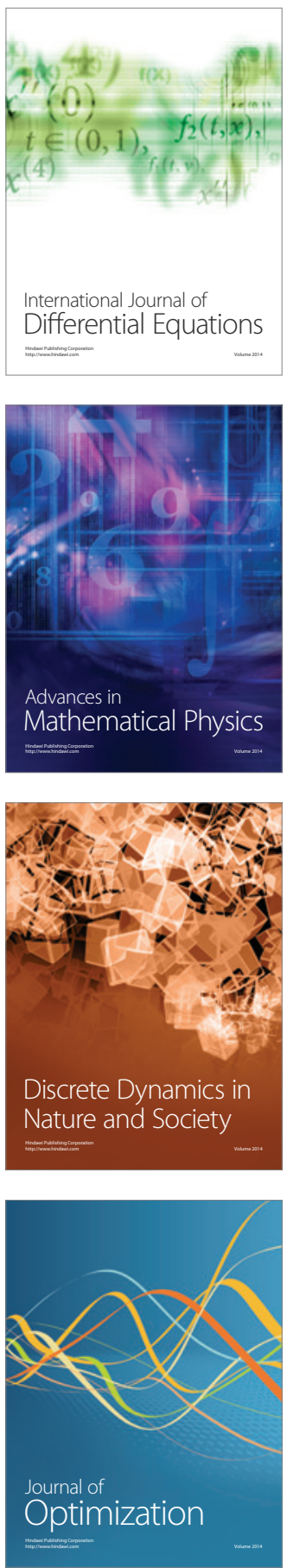